\title{
FUNCTIONAL MORPHOLOGY OF BIRDS’ BLOOD LEUKOCYTES
}

\section{Evgeniy Kolesnik ${ }^{1}$, Marina Derkho ${ }^{2}$, Victor Strizhikov ${ }^{2}$, Svetlana Strizhikova ${ }^{2}$, Tatyana Sereda ${ }^{2}$, Firdaus Gizatullina ${ }^{2}$, Maksim Rebezov $^{3,4^{*}}$}

\author{
${ }^{1}$ Department of General and Clinical Pathology, Faculty of Fundamental Medicine, Chelyabinsk State University, Chelyabinsk, Russian Federation \\ ${ }^{2}$ South Ural State Agrarian University, Troitsk, Russian Federation \\ ${ }^{3}$ Ural State Agrarian University, Yekaterinburg, Russian Federation \\ ${ }^{4}$ V.M. Gorbatov Federal Research Center for Food Systems of Russian Academy of Sciences, Moscow, Russian Federation \\ Received - September 23, 2020; Revision - November 14, 2020; Accepted - December 17, 2020 \\ Available Online December 15, 2020
}

DOI: http://dx.doi.org/10.18006/2020.8(Spl-2-AABAS).S374.S380

\section{KEYWORDS \\ Blood \\ Leukocytes \\ Heterophils \\ Eosinophils \\ Basophils \\ Lymphocytes \\ Chicken}

\begin{abstract}
The present research was carried out in an attempt to identify the problems associated with the morphofunctional analysis of vertebrate animal's leukocyte blood cells. For this blood samples of four age groups chickens (Gallus gallus L.) were collected and analysis was carried out as per the recommendations of the International Council for Standardization in Hematology for the identifying morpho-physiological characteristics of leukocytes of peripheral blood of birds. Results of the current study were based on the sample of early postnatal ontogenesis based on the analysis of high-resolution color microphotographs taken by the method of light-optical microscopy. Results of the current study revealed that the cells have the well-designed of all granular leukocytes typical "eosinophilic" nucleus with two segments. Sometimes cells contain polysegmental nuclei that are eccentrically located. Further, in contrast to heterophils, the eosinophilic nucleus has well-expressed contours, formed by irregularly shaped chromatin blocks with an optically denser structure. There are also eosinophils with stick-nuclei. The smallest blood granulocytes of the birds are basophils. Basophils differ in structure slightly basophilic color cytoplasm with intensively basophilic granules of rounded or other forms of different size. The sizes of granules in basophils are smaller than the other types of granular leukocytes. Thus, based on the analysis of qualitative color microphotographs of white blood cells of chickens of neonatal ontogenesis performed by optical microscopy, the differential morpho-physiological markers of leukocytic cells of peripheral blood of birds were marked and characterized.
\end{abstract}

* Corresponding author

E-mail: rebezov@yandex.ru (Maksim Rebezov)

Peer review under responsibility of Journal of Experimental Biology and Agricultural Sciences.

Production and Hosting by Horizon Publisher India [HPI] (http://www.horizonpublisherindia.in/).

All rights reserved.
All the articles published by Journal of Experimental Biology and Agricultural Sciences are licensed under a Creative Commons Attribution-NonCommercial 4.0 International License Based on a work at www.jebas.org.

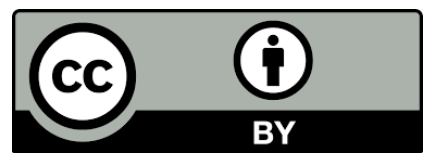




\section{Introduction}

The most important issues associated with the general blood cell morphology are the differentiation between the leukocyte cells of animals and humans (Maxwell et al., 1992; Clark et al., 2009; Campbell, 2015; Palmer et al., 2015; Lazarus \& Schmaier, 2019; Rodgers \& Young, 2019). Undoubtedly, there is a demand for objective criteria of functional morphological classification of white blood cell elements, which is crucial in all immune processes of the organism and health preservation, especially in avian (Faradilla et al., 2020).

Studying the cellular and humoral links of the immune process, the problems of finding differential morpho-physiological markers of blood corpuscles remain serious and somewhat non-clarify (Bolotnikov \&Solovyov, 1980; Campbell, 1994; Lazarus \&, Schmaier, 2019). A more important aspect of blood cell morphology is the fact that at present there is no unified approach in own differentiation of leukocytes of birds.

Gallus gallus (L.) chickens were used as a "biomedical model" during the study of the congenital immunity of vertebrate animals and "adaptive immune response" in general biology and veterinary medicine (Kaiser, 2010). In bird blood (Aves), the functional equivalent of mammals (Mammalia) neutrophils are mainly heterophils (Maxwell et al., 1992; Campbell, 1994; Maxwell \& Robertson, 1998; Chuammitri et al., 2009; Harvey, 2011; Campbell, 2015; Zhao et al., 2015; Bílková et al., 2017). Granulocytic phagocytes are an integral part of innate immune protection, with a primary role in the attack and killing of microorganisms (Maxwell \& Robertson, 1998; Chuammitri et al., 2009; Lazarus \& Schmaier, 2019; Rodgers \& Young, 2019). Specific features in the structure of peripheral blood of birds are granular leukocytes, which received a group name polymorphonuclear cells (PMNs) (Nesterova et al., 2015; Sharipova et al., 2017; Rodgers \& Young, 2019). PMNs help in the understanding synthetic group of granular white blood cells viz., neutrophils, eosinophils, and basophils with the different structural-spatial organization of the nucleus (Nesterova et al., 2015; Lazarus \& Schmaier, 2019; Rodgers \& Young, 2019). PMNs characterizes as the most general morphophysiological criteria, while the stage of the nucleus and cell development, i.e., the level of nuclear apparatus segmentation and cytoplasm organization, is derivative of the clinical link in the PMNs nomenclature (Clark et al., 2009; Palmer et al., 2015)

Polymorphonuclear leukocytes have been described and defined as "single celled secretory glands" by the World Health Organization (WHO) (Berezhnaya, 1988). While, Paul Ehrlich described neutrophils as "secretory cells." (Berezhnaya, 1988). The beginning of the formation and maintenance of immunological homeostasis is based on the phenomena of phagocytosis and secretion of various substances by neutrophils providing nonspecific immunity (Berezhnaya, 1988). Besides, as a result of subsequent work, mediator functions and modulation of the specific immunity link were included (Chuammitri et al., 2009; Kaiser, 2010; Nesterova et al., 2015; Kolesnik \& Derkho, 2018a; Lazarus \& Schmaier, 2019).

Recent works showed that blood granulocytes used a similar phagocytosis mechanism in different types of vertebrates including humans (Chuammitri et al., 2009; Kaiser, 2010; Nesterova et al., 2015; Kolesnik \&Derkho, 2018b). The leading ones are complex "neutrophil extracellular networks" integrating oxidative explosion, degradation, and decation of cytoplasmic granules with the releases of antimicrobial proteins, which as a result provide an effective performance of "cell-mediated adaptive immune response".

Thus, in recent years it was reported that nuclear proteins histones along with elastase, defensins, and other enzyme and non-enzyme cationic proteins from cytoplasmic granules of polymorphonuclear leukocytes (PMNs) form in vitro and in vivo "chicken heterophil extracellular traps" of congenital immunity in the bird's blood (Chuammitri et al., 2009; Kaiser, 2010; Kolesnik \& Derkho, 2018a). Further, Chuammitri et al. (2009) suggested that nowadays "heterophilic extracellular traps" are characterized as an important heterophilically mediated immune mechanism of protection in chickens (Chuammitri et al., 2009).

At the same time, in many respects, there are no unified approaches and algorithms in questions of morphophysiological analysis of the blood structure, clinical characteristics of the birds' blood form elements. There are significant differences in nomenclature and morphofunctional characterization of the blood picture of birds' blood in comparison with mammals. Further, the blood corpuscles of birds, in comparison with mammals, have significant morphological features. Only a few publications are available on the qualitative color microphotographs of bird blood cells obtained by the method of light-optical microscopy (Campbell, 1994; Maxwell \& Robertson, 1998; Clark et al., 2009; Harvey, 2011).

Considering this, the purpose of the current study was to reveal and characterizes differential morphophysiological markers of birds' blood leukocytes which was based on the morphofunctional analysis of microphotographs of peripheral blood cells of broiler chickens early on in life.

\section{Materials and Methods}

The current study was carried out as a part of the complex program of studying physiological adaptations of homeostasis of broiler chickens of neonatal ontogenesis, grown under the conditions of 
standardized industrial technologies (Kolesnik, 2017; Kolesnik \& Derkho, 2018a; Kolesnik \& Derkho, 2018b; Hubbard ISA Guide, http://hubbardbreeders.com/).

Blood smears of $1,7,23$, and 42 days of postnatal ontogenesis Hubbard F15 chickens (mark as P1, P7, P23, P42, and each group has $n=10$ ) were stained according to the Pappenheim method (Kolesnik \& Derkho, 2018a). Microphotographs of prepared smear were taken by large biological microscope ("MBB - 1A", "LOMO", Russia) with a micrographic eyepiece camera of a 5megapixel matrix (Full HD High resolution "HAYEAR" CMOS 5.0 Megapixel microscope video camera, PRC), with visualization, in the program "ToupView" ("ToupTek Photonics", PRC, http://www.touptek.com/). For the most qualitative image of blood cells, $90 \mathrm{X}$ oil immersion apochromatic lens with an aperture of 1.3 was used ("LOMO", Russia). The video camera was calibrated according to the scale of the object-micrometer for passing light with the scale division of $0.01 \mathrm{~mm}$ ("WMP" GHOST 7513 - 55 "LOMO", Russia). In the program "PhotoM 1.21" (Russia) (http://t-lambda.chat.ru/download.html, date of circulation January $12,2019)$ the following indicators were determined: $S_{k}-$ the area of a cell $\left(\mu \mathrm{m}^{2}\right) ; S_{y}$ - the area of a cell nucleus $\left(\mu \mathrm{m}^{2}\right) ; S_{c}$ - the area of cytoplasmic granules $\left(\mu \mathrm{m}^{2}\right)$. Nuclear cytoplasmic ratio (NSC), $\mathrm{NSC}=\mathrm{S}_{\mathrm{y}} / \mathrm{S}_{\mathrm{c}}$, where: $\mathrm{S}_{\mathrm{y}}-$ the area of the cell nucleus $\left(\mu \mathrm{m}^{2}\right) ; \mathrm{S}_{\mathrm{c}}-$ cytoplasmic granule area $\left(\mu \mathrm{m}^{2}\right)$.

The degree and reliability of differences for the obtained results were evaluated using a parametric T-test (single sample) in the program STATISTICA 8.0 ("StatSoft, Inc.", USA). The critical level of significance of the value difference was taken as $p \leq 0.05$.

\section{Results and discussion}

Bird lymphocytes (Aves) are smaller in size than mammals (Mammalia) (Kolesnik et al., 2019). Small, medium and large lymphocytes are distinguished in the peripheral blood of birds (Figure 1: 1.1 - 1.3, see table). Such cell division is based not only on different size of lymphatic agranulocytes but also on the values of their nuclear-protoplasmic ratio, the structure of nucleus chromatin, localization (orientation) of the nucleus in the cell, and coloring of protoplasm and nucleus (Figure 1-1.3; table 1). Small and medium lymphocytes have a small volume of the cytoplasm In small lymphocytes, the perinuclear zone is weakly expressed, in medium lymphocytes it is larger, but also poorly visible. The nucleus of small lymphocytes looks hyperchromic, which is quite reasonable, given the regularities of maturation of non-grain leukocytes from large to mature small. The picture of the surface of nuclear chromatin is small and medium lymphocytes resembles the "lunar surface", it is painted intensely basophilic (basichromatin), especially in small cells, structured (in comparison with homogeneous, in the nucleus of mammalian lymphocytes), dense, forms irregularly shaped blocks (Figure 1:
1.3). Large lymphocytes are represented by cells with well developed pericarion (Fig. 1: 1.1). Nuclei are located eccentrically, at one of the cell poles. The shape of lymphocyte nuclei is more often rounded but may be slightly rounded in small and medium forms, the nuclei may have a slight intussusception of cariolemma.

The nuclei contain well structured heterogeneous chromatin, which looks like blocks of different sizes and irregular shapes. The chromatin is basophilic but has a comparatively lighter shade than that of small and medium lymphocytes (Figure 1: 1.1). Large lymphocytes with a small volume of cytoplasm and a centrally located large, pycnotic nucleus with a well-expressed large lumpy chromatin are also found in the blood picture of birds (Figure 1: 1.2). The chromatin of these lymphocytes is compact, grooved, and intensively basophilic (Figure 1: 1.2, 1.3). The cytoplasm of all forms of lymphocytes is well structured (in contrast to the white blood cells of mammals), is slightly basophilic, evenly colored, but has an excellent hue in lymphocytes with varying degrees of the perinuclear zone (Figure 1: 1.1 - 1.3).

Plasmocytes (synonyms: plasma cells, reactive lymphocytes, Türk cells) are often found in the blood picture of birds, unlike mammalian vertebrates. Plasmocytes are large and medium-sized lymphocytes with intermittently basophilic (ultramarine) cytoplasm with well-detectable perinuclear zone and eccentrically localized round nucleus (Figure 1: 1.4). Among all large lymphocytes, the darkest colored nucleus is formed by Türk cells; chromatin in them is formed by many small basophilic colored blocks, which contain narrow grooves of oxychromatin colored pink. The cytoplasm of reactive lymphocytes is distinguished by a dark blue gradient transition along the edges (perimeter) of the cell (Figure 1: 1.4)

The largest forms of agranulocytes in bird blood are monocytes (synonyms: mononuclear, macrophages). Monocytes, among agranular leukocytes, are distinguished by peculiarities of nuclearprotoplasmic ratio, with the largest shift towards the volume of cytoplasm and pleomorphism (polymorphism) of nuclei (Figure 1: 1.5, 1.6, table 1).

In the nucleus presence of the heterochromatin can be seen, which has a large or finely bumped structure, and forms a network-like pattern. Sometimes optically denser and larger sections of heterochromatin together with smaller blocks form a mosaic reticular structure of the monocyte nucleus (Figure 1: 1.5, 1.6). Further, monocytes usually have a rounded shape, with a centrally or eccentrically located polymorphous nucleus, while due to phagocytosis and the formation of wide pseudoids, macrophages acquire an irregular molten form. The cytoplasm of monocytes is poorly basophilic colored, well structured, has a slightly foamy appearance, and contains small vacuoles of various shapes and sizes (Figure 1: 1.5, 1.6). 

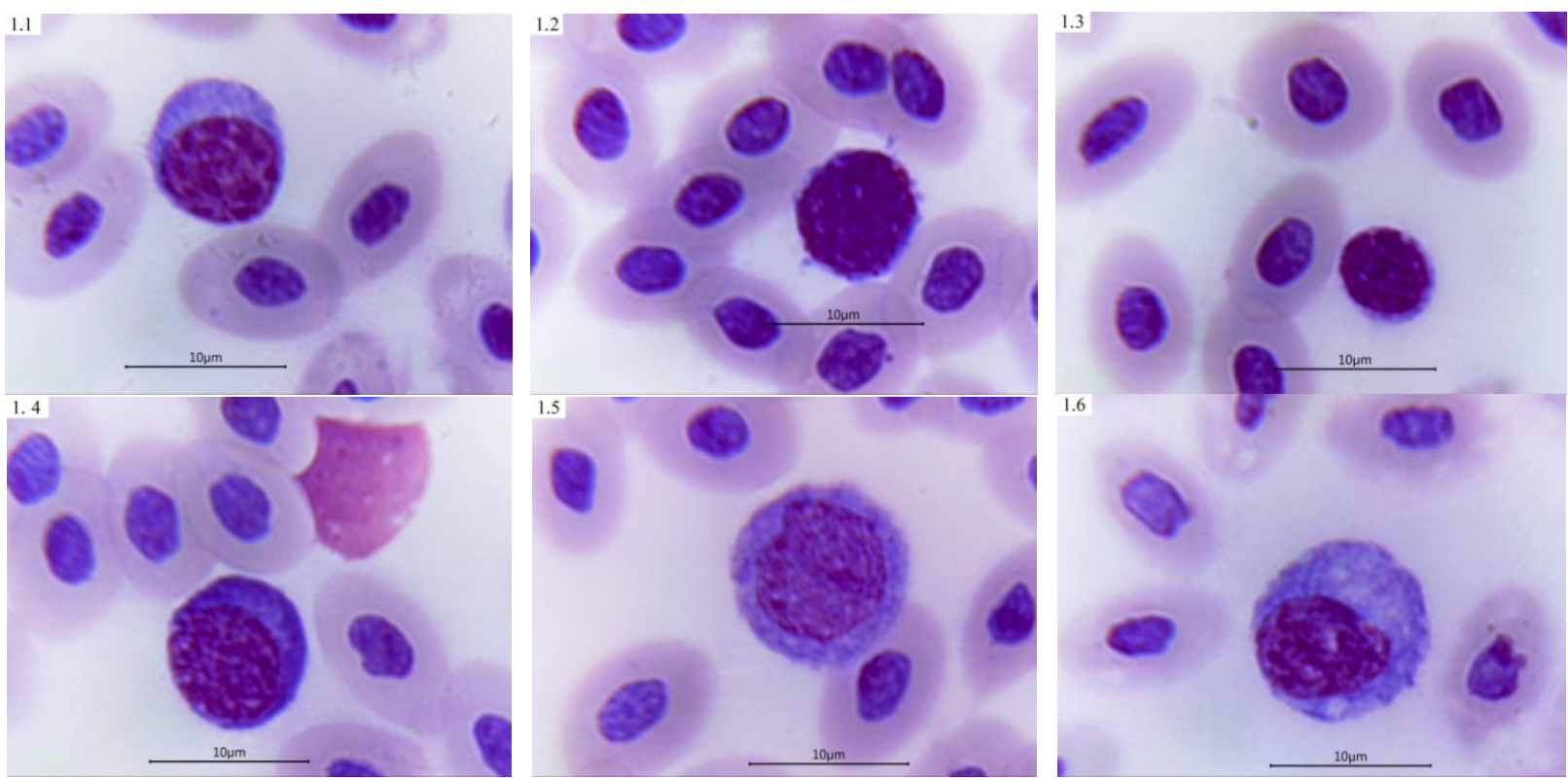

Figure 1 Peripheral blood of broiler chickens (age of birds is given in brackets), agranulocytes: 1.1 wide plasma (1st day); 1.2 narrow plasma (42nd day) - large lymphocytes; 1.3 (42nd day) - small lymphocytes; 1.4 (42nd day) - plasmocyte; 1.5 (42nd day) and 1.6 (23rd day) monocytes.

Table 1 - Morphologic features of leukocytes Hubbard ISAF15 ( $\mathrm{n}=5)$

\begin{tabular}{|c|c|c|c|c|}
\hline \multirow{3}{*}{ Indicator } & \multicolumn{4}{|c|}{ Size of cell structures } \\
\hline & \multicolumn{4}{|c|}{ Agranulocytes } \\
\hline & $\mathrm{S}_{\mathrm{K}}\left(\mu \mathrm{m}^{2}\right)$ & $S_{s}\left(\mu m^{2}\right)$ & $\begin{array}{c}\text { nuclear- } \\
\text { cytoplasmic ratio }\end{array}$ & $\mathrm{S}_{\mathrm{r}}\left(\mu \mathrm{m}^{2}\right)$ \\
\hline Large wide-plasma lymphocytes & $66.03 \pm 1.65^{*}$ & $40.26 \pm 3.27 *$ & $1.56 \pm 0.03^{*}$ & - \\
\hline Large narrow plasma lymphocytes & $52.93 \pm 1.47$ & $43.96 \pm 3.18$ & $4.90 \pm 0.09$ & - \\
\hline Medium lymphocytes & $40.70 \pm 3.18 *$ & $28.27 \pm 1.98 *$ & $2.27 \pm 0.11 *$ & - \\
\hline Small lymphocytes & $28.29 \pm 2.07$ & $22.58 \pm 1.74$ & $3.95 \pm 0.09$ & - \\
\hline Monocytes & $105.26 \pm 2.21 *$ & $57.86 \pm 1.30 *$ & $1.22 \pm 0.07 *$ & - \\
\hline \multicolumn{5}{|c|}{ Granulocytes } \\
\hline Heterophiles & $68.36 \pm 2.03 *$ & - & - & $0.84 \pm 0.03 *$ \\
\hline Eosinophiles & $77.85 \pm 2.91^{*}$ & - & - & $0.33 \pm 0.01 *$ \\
\hline Bazophiles & $64.51 \pm 1.22 *$ & - & - & $0.89 \pm 0.03 *$ \\
\hline
\end{tabular}

Granular leukocytes are evolutionarily the first link of immune reactions in the vertebrate organisms (Bolotnikov \& Solovyov, 1980). The most intensive and very transient metabolic processes of granular leukocytes provide an immediate allergic and inflammatory response, primary detoxification reactions, and phagocytosis at the microphage level in the internal environment of the body; in this connection, the circulation period of granulocytes is no more than 8 - 15 days (Bolotnikov \& Solovyov, 1980; Maxwell \& Robertson, 1998). The marked functional characteristics of granular form elements also determine morphological features of granulocytes, observed in a bird blood smear picture.

Heterophils (a form of neutrophils in birds, an outdated synonym: pseudoeoesinophils), morphologically, differ from the typical neutrophils of most mammal species, characteristics of the granular cytoplasmic apparatus, and peculiarities of the nucleus. According to the authors' figurative expression (Maxwell \& Robertson, 1998), the morphofunctional characteristic of blood heterophils is a "window to the state of bird health". 
Mature heterophyll is a segmental leukocyte with an eccentric nucleus consisting of two or three segments and a cytoplasm containing numerous, mainly stick-shaped, as well as spindleshaped, sometimes rounded and oval cytoplasmic granules (Figure $2: 2.1,2.2$, table). The abundant granularity can partially mask the contours of the nucleus, resulting in visually difficult to determine the stage of segmentation of the nucleus. "Neutrophilic" or amphofilic color of the cytoplasmic matrix of typical mammalian neutrophils is also typical for cytoplasmic granules of heterophils of birds. Thus, heterophyll grains are dark pink and may have a small lilac shade. Heterophyll cytoplasm may be slightly basophilic or be almost colorless, sometimes slightly oxyphilic (acidophilic) (Figure 2: 2.1, 2.2). The heterophyll nucleus is more pycnotic than that of typical mammalian neutrophils, with wellstructured heterochromatin formed by boulders colored in light purple with a dark lilac shade.

The chromatin blocks of the heterophiles, morphologically - in their structure and shape, are more similar to nuclear nuclei, as they have a more correct oval and rounded shape, compared to the blocks of typical irregular shape, typical for lymphocyte nuclei (Fig. 1: $1.1-1.3$ \& Figure 2: 2.1, 2.2).

Eosinophils of birds are the least specific in structure. These are segmental-nucleus granulocytes having characteristic round form, cytoplasmic granules of similar shape and size, with expressed oxyphilic coloring with saturated orange tint (Figure 2: 2.3, 2.4, table 1). In comparison with heterophils, the cytoplasm is always well visualized, with weak basophilic coloring typical for eosinophils (Figure 2: 2.3, 2.4). In this case, the cytoplasm of cells is often clearly structured (Figure 2: 2.3).

The cells have the well-designed of all granular leukocytes typical "eosinophilic" nucleus with two segments (Figure 2: 2.3). Sometimes cells contain polysegment nuclei that are eccentrically located (Figure 2: 2.4). In contrast to heterophils, the eosinophilic nucleus has well expressed contours, formed by irregularly shaped chromatin blocks with optically denser structures (Figure 2: 2.1, $2.2,2.3,2.4)$.

The smallest blood granulocytes of the birds are basophils. Basophils differ in structure and have slightly basophilic colored cytoplasm with intensively basophilic granules of rounded or other forms of different size (Figure 2: 2.5, 2.6, table 1). The number of granules in basophils is much smaller than the other types of granular leukocytes (Figure 2: 2.1 - 2.4, \&2.6, table 1).

In the publications of Campbell (1994), a description of bird basophils is available; whose graininess in many respects masks the contours of core segmentation. However, in the blood smears of chickens, the severity of nucleus contours was much less marked than in heterophils, although the number of granules in the cytoplasm was not large enough to mask nucleus segmentation (Figure 2: 2.5, 2.6). The camouflage of nucleus segmentation is primarily related to the weak structure of chromatin. It may be noted that bird basophils have the most "delicate" chromatin structure in the nucleus among other granulocyte species (Figure 2: $2.5,2.6)$. The morphological peculiarity of basophils is also similar to the color shade of cytoplasm and nucleus, which is in agreement with the similar data given by Bolotnikov \& Solovyov (1980).
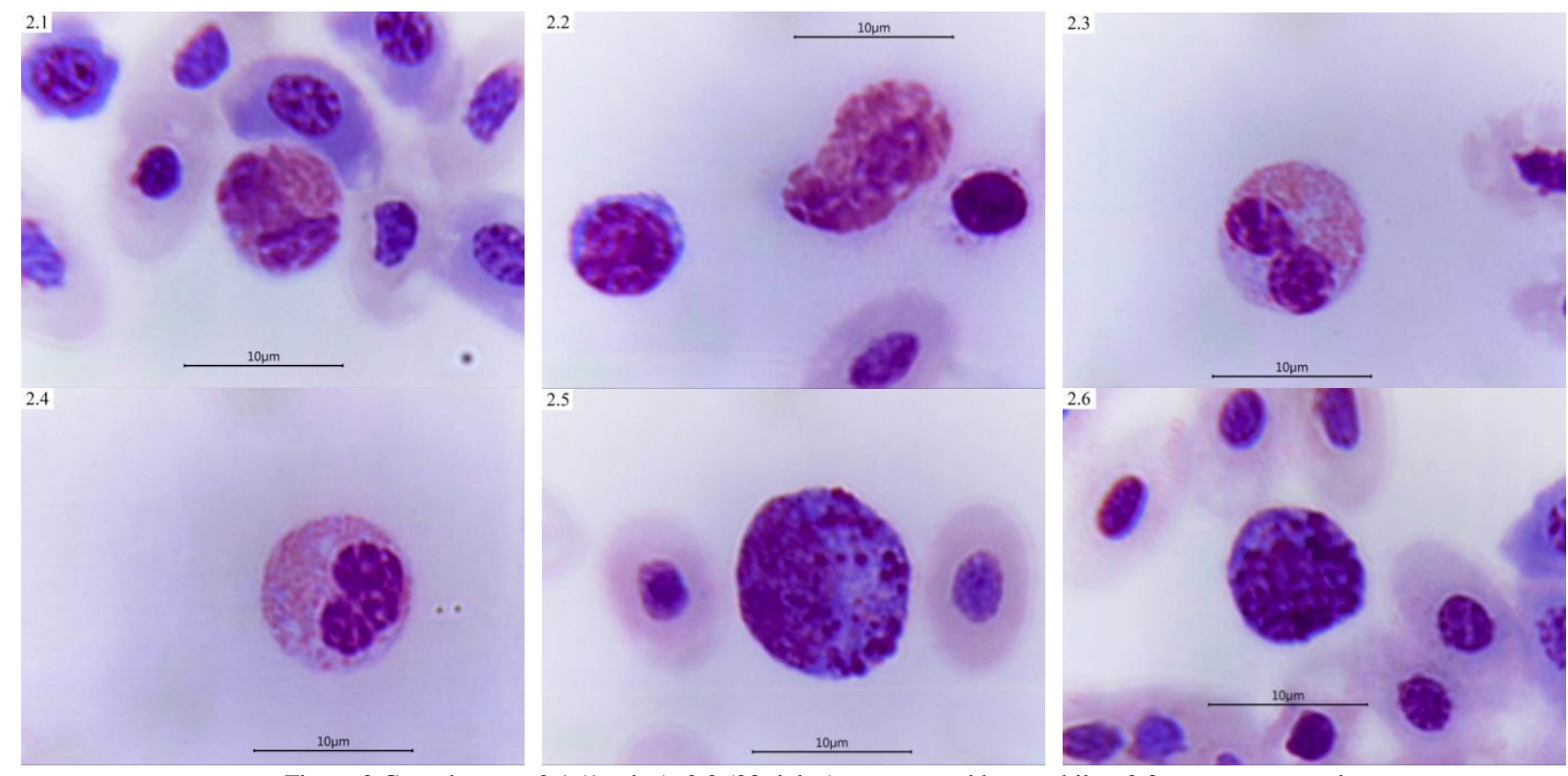

Figure 2 Granulocytes: 2.1 (1st day); 2.2 (23rd day) - segmented heterophiles; 2.3 - two-segmented; 2.4 - polysegmented (23rd day) - eosinophiles; 2.5 - basophilic metamyelocyte and 2.6 - basophile: (1st day).

Journal of Experimental Biology and Agricultural Sciences http://www.jebas.org 
Single myelocytic forms of basophils are found in the blood of birds. These are different in the cytoplasmic granules in three ways such as (i) a relatively small amount of grains in the protoplasm of cells, (ii) characterized by the different sizes of granules, (iii) by their metachromasia, i.e., the presence of a small number of grains with pink-violet shades (Figure 2: 2.5).

\section{Conclusion}

Thus, based on the analysis of qualitative color microphotographs of white blood cells of chickens of neonatal ontogenesis performed by optical microscopy, differential morphophysiological markers of leukocytic cells of peripheral blood of birds were marked and characterized. These findings will be helpful to future studied on avian hematology.

\section{Conflict of Interest}

Authors would hereby like to declare that there is no conflict of interests that could possibly arise.

\section{Reference}

Berezhnaya NM (1988) Neutrophils and Immunological homeostasis, Naukova Dumka Publishing House, Kiev. Pp. 192.

Bílková B, Bainová Z, Janda J, Zita L, Vinkler M (2017) Different breeds, different blood: Cytometric analysis of whole blood cellular composition in chicken breeds. Veterinary Immunology and Immunopathology 188:71-77.

Bolotnikov IA, Solovyov YuV (1980) Hematology of birds, Publishing house "Nauka", Leningrad, Pp. 116.

Campbell TW (2015) Exotic animal hematology and cytology. 4th edition, Wiley-Blackwell. A John Wiley \& Sons, Ltd., Publication, Ames, Iowa, Pp. 403.

Campbell TW (1994) Hematology. In: Ritchie BW, Harrison GJ, Harrison LR (Eds.) Avian medicine: Principles and applications. Wingers Publishing, Inc. Lake Worth, Florida, Pp. 176-198.

Chuammitri P, Ostojić J, Andreasen CB, Redmond SB, Lamont SJ, Palić D (2009) Chicken heterophil extracellular traps (HETs): novel defense mechanism of chicken heterophils. Veterinary Immunology and Immunopathology 129(1-2):126-131.

Clark P, Boardman W, Raidal S (2009) Atlas of clinical avian hematology. John Wiley \& Sons Pp. 8.

Faradilla ZSH, Yunus M, Hermadi HA (2020) The effect of dietary administration of virgin coconut oil on differential leukocytes in infected chicken with Eimeria tenella. Journal of World's Poultry Research 10(4): 615-622.
Harvey JW (2011) Veterinary Hematology-E-Book: A Diagnostic Guide and Color Atlas. Elsevier Health Sciences.

Kaiser P (2010) Advances in avian immunology—prospects for disease control: a review. Avian Pathology 39(5):309-324.

Kolesnik EA, Derkho MA (2018a) About participation of pituitary-adrenocortical hormones in regulation of blood cellular pool in chicken-broilers. Problems of Productive Animal Biology 1:64-74.

Kolesnik E.A., Derkho M.A., Lebedeva I.A. Comprehensive morphophysiological description of the immune lysosomal cationic protein of leukocytes in the early ontogeny of broiler chickens. UchenyeZapiskiKazanskogoUniversiteta.

SeriyaEstestvennyeNauki, 2019, vol. 161, no. 3, pp. 440-458. doi: 10.26907/2542-064X.2019.3.440-458. (In Russian).

Kolesnik EA, Derkho MA (2018b) Immune cationic protein of neutrophils as a factor of nonspecific resistance and physiological basis for the development of probiotics. In: Microbial technologies in poultry farming and animal husbandry: a collection of abstracts of the All-Russian scientific and practical conference). Institute of Fundamental medicine and biology, Kazanskij universitet Publ., Kazan (Volga Region) Federal university, Kazan Pp.18.

Kolesnik EA (2017) Algorithm of the analysis of elements constituting the factor model of the humoral regulation of the metabolism in broiler chicken. Bulletin of Tver state university. Series: Biology and Ecology 1:69-75.

Lazarus HM, Schmaier AH (2019) Concise Guide to hematology. 2th edition, Springer, Cham, Switzerland Pp. 542.

Maxwell MH, Robertson GW, Mitchell MA, Carlisle AJ (1992) The fine structure of broiler chicken blood cells, with particular reference to basophils, after severe heat stress. Comparative Haematology International 2(4):190-200.

Maxwell MH, Robertson GW (1998) The avian heterophil leucocyte: a review. World's poultry Science Journal 54(2):155178.

Nesterova IV, Kolesnikova NV, Chudilova GA, Lomtatidze LV, Kovaleva SV, Evglevsky AA (2015) Neutrophilic granulocytes: a new look at the "old players" on the immunological field. Immunology 36(4):257-265.

Palmer L, Briggs C, Mcfadden S, Zini G, Burthem J, Rozenberg G, Proytcheva M, Machin SJ (2015) ICSH recommendations for the standardization of nomenclature and grading of peripheral blood cell morphological features. International Journal of Laboratory Hematology 37(3):287-303. 
Rodgers GP, Young NS (2019) The Bethesda handbook of clinical hematology. 4th edition, Lippincott Williams \& Wilkins / Wolters Kluwer, Philadelphia, Baltimore, New York, London, Buenos Aires, Hong Kong, Sydney, Tokyo, Pp. 862 p.

Sharipova A, Khaziev D, Kanareikina S, Kanareikin V, Rebezov M, Okuskhanova E, Suychinov A, Yessimbekov Z (2017) The effects of a probiotic dietary supplementation on the livability and weight gain of broilers. Annual Research and Review in Biology 19(6): 1-5.
The International Council for standardization in haematology (ICSH) [Electronic resource]. International Council for standardization in haematology: [site]. 2019. URL: https://icsh.org/.

Zhao W, Liu J, Xu R, Zhang C, Pang Q, Chen X, Liu S, Hong L, Yuan J, Li X, Chen Y (2015) The gametocytes of Leucocytozoon sabrazesi infect chicken thrombocytes, not other blood cells. PloS one 10(7):p.e0133478.

Journal of Experimental Biology and Agricultural Sciences http://www.jebas.org 